\title{
Gauge Theories and non-Commutative Geometry
}

\section{A review}

John lliopoulos ${ }^{1, a}$

${ }^{1}$ ENS Paris

\begin{abstract}
This is a very brief report on the attempts to introduce the concepts of noncommutative geometry in the theoretical description of the fundamental interactions. A particular emphasis will be given to gauge theories. A large part of the report is based on some on-going work in collaboration with Prof. Manolis Floratos. The main results have already been published[1], but there are many open problems remaining.
\end{abstract}

\section{Introduction}

There may be several motivations to study a quantum theory in a space with non-commutative geometry and I will list some of them here.

- Not surprisingly, the first proposal goes back to W. Heisenberg who, in a letter to Peierls in 1930[2], suggested that non-commutativity among space coordinates could eliminate the short distance singularities. He tried to convince Peierls and Pauli to work on this problem, but, apparently, Pauli did not think much of the idea. ${ }^{1}$ He talked instead to Oppenheimer[4] who gave it as a problem to his PhD student H.S. Snyder. Snyder published a paper in 1947[5] in which he tries to enlarge the quantum mechanical commutation relations to a system involving the commutators among space components. His main concern seems to have been compatibility with Poincaré invariance. He wrote a system of rather obscure commutation relations of the form:

$$
\begin{aligned}
{[x, y] } & =\left(\mathrm{i} a^{2} / \hbar\right) L_{z} & {[t, x] } & =\left(\mathrm{i} a^{2} / \hbar c\right) M_{x} \\
{\left[x, p_{x}\right] } & =\mathrm{i} \hbar\left[1+(a / \hbar)^{2} p_{x}^{2}\right] & {\left[x, p_{y}\right] } & =\mathrm{i} \hbar(a / \hbar)^{2} p_{x} p_{y}
\end{aligned}
$$

where $a$ is the parameter defining space non-commutativity and $L$ and $M$ are the generators of Lorentz transformations. As far as I know neither Snyder nor anybody else attempted to write a field theory based on these relations and there was no follow up for many years. ${ }^{2}$ Snyder himself left noncommutative geometry and had a successful career as an accelerator engineer[6]. In fact, as history evolved, Pauli was probably right. The motivation based on short distance singularities did not prove fruitful for elementary particle physics. With the development of the renormalisation programme in the framework of quantum field theories, the problem of ultraviolet divergences took a completely different turn. While a space cut-off makes all theories finite, the renormalisation programme applies

\footnotetext{
ae-mail: ilio@lpt.ens.fr

${ }^{1}$ In a letter to Bohr he commented: “....it seems to be a failure for reasons of physics.”[3]

${ }^{2}$ It is also strange that Oppenheimer's name is never cited in the paper.
} 
to very few and very specific field theories. It is a most remarkable fact that they are precisely the ones chosen by Nature. It is not finiteness but rather lack of sensitivity to unknown physics at very short distances that turned out to be the important criterion. The geometry of physical space may still produce an ultraviolet cut-off, but its presence is not relevant for the calculation of physical processes among elementary particles.

- However, almost at the same time, a new motivation for studying theories in a non-commutative space appeared, although only recently it was fully appreciated. In 1930 L.D. Landau[7] solved the problem of the motion of an electron in an external constant magnetic field and, besides computing the energy levels, the so-called "Landau levels", he showed that the components of the velocity operator of the electron do not commute. A simple way to visualise this result is to think of the classical case where the electron follows a spiral trajectory whose projection on a plane perpendicular to the field is a circle. In Landau's quantum mechanical solution the centre's coordinates are:

$$
x_{c}=\frac{c p_{y}}{e H}+x \quad ; \quad y_{c}=-\frac{c p_{x}}{e H}
$$

which shows that the two coordinates do not commute. The magnetic field has induced a noncommutative structure on space itself. Following Heisenberg's suggestion, R. Peierls[8] showed that, at least the lowest Landau level, can be obtained by using this space non-commutativity. Since the presence of non-vanishing magnetic-type external fields is a common feature in many modern supergravity and string models, the study of field theories formulated on spaces with non-commutative geometry[9] has become quite fashionable.

- A new element was added a few years ago with the work of N. Seiberg and E. Witten[10] who showed the existence of a map between gauge theories formulated in spaces with commuting and non-commuting coordinates.

- An independent line of approach has been initiated by A. Connes[9][11] and co-workers and aims at constructing a gauge theory with spontaneously broken symmetry using the techniques of non-commutative geometry. The result which relates the symmetry breaking parameter to the distance between different branes has been first obtained in this approach.

- A different but related motivation comes from $S U(N)$ gauge theories at large $N$ and matrix models.

In this report I will present mostly the last point with some remarks on Connes' approach at the end. There have been many review articles, both from the mathematics as well as the physics point of view, and an incomplete list is given in reference[12].

\section{Some elementary formulae}

Space non-commutativity means that we have a commutation relation of the form:

$$
\left[x_{\mu}, x_{v}\right]=i \theta_{\mu \nu}
$$

In the simplest case, the one we will consider in this talk, $\theta$ is constant (canonical, or Heisenberg case). Other choices have also been considered: $\left[x_{\mu}, x_{\nu}\right]=i F_{\mu \nu}^{\rho} x_{\rho}$ (Lie algebra case, the one analysed by Snyder), or $x_{\mu} x_{v}=q^{-1} R_{\mu \nu}^{\rho \sigma} x_{\rho} x_{\sigma}$, (sometimes called quantum space case).

We also define the derivative:

$$
\partial^{\mu} x_{v}=\delta_{v}^{\mu} \Rightarrow\left[x_{\mu}, f(x)\right]=i \theta_{\mu \nu} \partial^{v} f(x)
$$

and $\mathrm{a} *$ product which is formally given by:

$$
f * g=\left.e^{\frac{i}{2} \frac{\partial}{x_{\mu}} \theta_{\mu \nu} \frac{\partial}{y_{\nu}}} f(x) g(y)\right|_{x=y}
$$


All computations can be viewed as expansions in $\theta$, which, in the Landau paradigm, is proportional to the inverse of the external magnetic field. It is not clear whether any more efficient computational tools can be developed which would take full advantage of the non-commutative nature of space and this seems to be a severe limitation of the power of the approach. We do not have a quantum field theory in a space with non-commutative geometry which is not an expansion around ordinary space. This may be the reason why most formulations of gauge theories seem to be limited to the classical theory and it is not easy to extend to them the BRST symmetry.

The main remark which underlines our approach is the following: Let $\phi^{i}(x) i=1, \ldots, N N \rightarrow \infty$ be an $N$-component field defined in a $d$-dimensional space. At large $N$ we can write $\phi^{i}(x) \rightarrow \phi(\sigma, x)$ $0 \leq \sigma \leq 2 \pi$, i.e. we can consider $\phi^{i}(x)$ as the Fourier components of a field in $d+1$ dimensions with the extra dimension being a circle. In this case we have:

$$
\sum_{i=1}^{\infty} \phi^{i}(x) \phi^{i}(x) \rightarrow \int_{0}^{2 \pi} \mathrm{d} \sigma(\phi(\sigma, x))^{2}
$$

However, the interaction term will be non local in $\sigma$ :

$$
\phi^{4} \rightarrow\left(\int_{0}^{2 \pi} \mathrm{d} \sigma(\phi(\sigma, x))^{2}\right)^{2}
$$

The crucial remark is that, for a Yang-Mills field $A_{\mu}(x)$ belonging to the adjoint representation of an $S U(N)$ group, the resulting expression at large $N$ is local[1]. Written explicitly we have:

Given an $S U(N)$ Yang-Mills theory in a $d$-dimensional space $A_{\mu}(x)=A_{\mu}^{a}(x) t_{a}$, there exists a reformulation in $d+2$ dimensions $A_{\mu}(x) \rightarrow \mathcal{A}_{\mu}\left(x, \sigma_{1}, \sigma_{2}\right)$ and $F_{\mu \nu}(x) \rightarrow \mathcal{F}_{\mu \nu}\left(x, \sigma_{1}, \sigma_{2}\right)$ with $\sigma_{1}$ and $\sigma_{2}$ appropriately chosen coordinates on a compact 2-dimensional surface, such that, at $N \rightarrow \infty$, the matrix commutators become the usual Poisson brackets with respect to $\sigma_{1}$ and $\sigma_{2}$.

$$
\begin{aligned}
{\left[A_{\mu}(x), A_{v}(x)\right] } & \rightarrow\left\{\mathcal{A}_{\mu}\left(x, \sigma_{1}, \sigma_{2}\right), \mathcal{A}_{v}\left(x, \sigma_{1}, \sigma_{2}\right)\right\} \\
{\left[A_{\mu}(x), \Omega(x)\right] } & \rightarrow\left\{\mathcal{A}_{\mu}\left(x, \sigma_{1}, \sigma_{2}\right), \Omega\left(x, \sigma_{1}, \sigma_{2}\right)\right\}
\end{aligned}
$$

where $\Omega$ is an element of the gauge group. We can show that the Yang-Mills action becomes:

$$
\int \mathrm{d}^{4} x \operatorname{Tr}\left(F_{\mu \nu}(x) F^{\mu v}(x)\right) \rightarrow \int \mathrm{d}^{4} x \mathrm{~d} \sigma_{1} \mathrm{~d} \sigma_{2} \mathcal{F}_{\mu v}\left(x, \sigma_{1}, \sigma_{2}\right) \mathcal{F}^{\mu v}\left(x, \sigma_{1}, \sigma_{2}\right)
$$

The $S U(N)$ gauge invariance has become invariance under area preserving diffeomorphisms of the 2-dimensional surface spanned by $\sigma_{1}$ and $\sigma_{2}$. The proof of this statement is essentially algebraic. A direct way[13] is to prove that at the limit $N \rightarrow \infty$ the $S U(N)$ structure constants, appropriately rescaled, go to those of [SDiff 2 . We can also show it explicitly for the sphere[1] and the torus[1][14]

A final remark: The equivalence (9) is established for the classical theories. To go to the quantum theory we should first find a suitable gauge and this can be done. But then we are facing a second problem: The quadratic part of the new 6-dimensional action has no derivatives with respect to the variables $\sigma_{1}$ and $\sigma_{2}$. As a result, the perturbation expansion cannot be defined. This is not surprising. In proving (9) we have not imposed 't Hooft's rescaling condition in which $g^{2} N$ is kept fixed and we recover, already at lowest order, the infinite number of graphs. It is possible, although we have no explicit proof, that we can absorb these divergences in a clever renormalisation scheme, but it is not clear whether any new insight can be obtained this way. The 4-dimensional theory we started from is renormalisable for any finite $N$. A different approach would be to expand around a non-trivial solution which, hopefully, captures part of the non-perturbative dynamics of the Yang-Mills theory. Such a "master field" has not yet been found. 


\section{Finite $N$ and non-commutative geometry}

The previous results were valid at $N \rightarrow \infty$. In this section I want to point out that we can extend them to any value of $N$ with the price of introducing non-commutative geometry for the extra 2-dimensional space[1]. I will sketch the proof for a "fuzzy" sphere and a "fuzzy" torus.

\subsection{A fuzzy sphere}

For the classical sphere a convenient choice of coordinates is given by the usual angles $\theta$ and $\phi$. We can write $x_{1}=\cos \phi \sin \theta, x_{2}=\sin \phi \sin \theta$ and $x_{3}=\cos \theta$. The corresponding spherical harmonics are given by:

$$
Y_{l, m}(\theta, \phi)=\sum_{\substack{i_{k}=1,2,3 \\ k=1, \ldots, l}} \alpha_{i_{1} \ldots i_{l}}^{(m)} x_{i_{1}} \ldots x_{i_{l}}
$$

where $\alpha_{i_{1} \ldots i_{l}}^{(m)}$ is a symmetric and traceless tensor. For fixed $l$ there are $2 l+1$ linearly independent tensors $\alpha_{i_{1} \ldots i_{l}}^{(m)}, m=-l, \ldots, l$.

We choose, inside $S U(N)$, an $S U(2)$ subgroup $^{3}$ whose generators we call $S_{i}$. They satisfy the commutation relations: $\left[S_{i}, S_{j}\right]=\mathrm{i} \epsilon_{i j k} S_{k}$. We can use them as a basis to build the $N^{2}-1$ generators of $S U(N)$ in the fundamental representation:

$$
S_{l, m}^{(N)}=\sum_{\substack{i_{k}=1,2,3 \\ k=1, \ldots, l}} \alpha_{i_{1} \ldots i_{l}}^{(m)} S_{i_{1}} \ldots S_{i_{l}} \Rightarrow\left[S_{l, m}^{(N)}, S_{l^{\prime}, m^{\prime}}^{(N)}\right]=\mathrm{i} f_{l, m ; l^{\prime}, m^{\prime}}^{(N) l^{\prime \prime}, m^{\prime \prime}} S_{l^{\prime \prime}, m^{\prime \prime}}^{(N)}
$$

where the constants $f^{(N)}$ are the $S U(N)$ structure constants in a somehow unusual notation. It is now clear that the three $S U(2)$ generators $S_{i}$, rescaled by a factor proportional to $1 / N$, will have well-defined limits as $N$ goes to infinity:

$$
S_{i} \rightarrow T_{i}=\frac{2}{N} S_{i} \text { implies }\left[T_{i}, T_{j}\right]=\frac{2 \mathrm{i}}{N} \epsilon_{i j k} T_{k} \text { and } T^{2}=T_{1}^{2}+T_{2}^{2}+T_{3}^{2}=1-\frac{1}{N^{2}}
$$

In other words: under the norm $\|x\|^{2}=\operatorname{Tr} x^{2}$, the limits as $N$ goes to infinity of the generators $T_{i}$ are three objects $x_{i}$ which commute and are constrained by $x_{1}^{2}+x_{2}^{2}+x_{3}^{2}=1$. This in turn shows that the classical Yang-Mills theory becomes the theory invariant under area preserving diffeomorphisms of equation (9) with the closed surface being a sphere $S^{2}$.

So much for the large $N$ limit. For any value of $N$, we can parametrise the three operators $T_{i}$ in terms of two operators, $z_{1}$ and $z_{2}$ as follows: ${ }^{4}$

$$
\begin{gathered}
T_{+}=T_{1}+\mathrm{i} T_{2}=e^{\frac{\mathrm{i} z_{1}}{2}}\left(1-z_{2}^{2}\right)^{\frac{1}{2}} e^{\frac{\mathrm{i} z_{1}}{2}} \\
T_{-}=T_{1}-\mathrm{i} T_{2}=e^{-\frac{\mathrm{i} z_{1}}{2}}\left(1-z_{2}^{2}\right)^{\frac{1}{2}} e^{-\frac{\mathrm{i} z_{1}}{2}} \\
T_{3}=z_{2}
\end{gathered}
$$

Then it is straightforward algebra[1] to prove the following algebraic statement:

$$
\left[z_{1}, z_{2}\right]=\frac{2 i}{N} \Leftrightarrow\left[T_{i}, T_{j}\right]=\frac{2 \mathrm{i}}{N} \epsilon_{i j k} T_{k}
$$

\footnotetext{
${ }^{3}$ To be precise, it must be the principal $S U(2)$ subgroup[15]. In practice this means that we must choose a suitable basis in the $S U(N)$ Lie algebra such that the generators of the chosen $S U(2)$ are represented in the standard way by $N \times N$ matrices forming an irreducible representation.

${ }^{4}$ A similar parametrisation has been used by T. Holstein and $\mathrm{H}$. Primakoff in terms of creation and annihilation operators[16].
} 
in other words, if $z_{1}$ and $z_{2}$ satisfy the Heisenberg algebra, the operators $T_{i}$ satisfy the $S U(2)$ algebra and the opposite is also true, the $S U(2)$ algebra for the operators $T_{i}$ imply the Heisenberg algebra among $z_{1}$ and $z_{2}$. From that point we can go on and show a formal equivalence:

Given an $S U(N)$ Yang-Mills theory in a $d$-dimensional space, there exists a reformulation in $d+2$ dimensions in which

$$
A_{\mu}(x) \rightarrow \mathcal{A}_{\mu}\left(x, z_{1}, z_{2}\right) \quad F_{\mu \nu}(x) \rightarrow \mathcal{F}_{\mu \nu}\left(x, z_{1}, z_{2}\right) \text { and }\left[z_{1}, z_{2}\right]=\frac{2 i}{N}
$$

such that

$$
\begin{aligned}
{\left[A_{\mu}(x), A_{v}(x)\right] } & \rightarrow\left\{\mathcal{A}_{\mu}\left(x, z_{1}, z_{2}\right), \mathcal{A}_{v}\left(x, z_{1}, z_{2}\right)\right\}_{\text {Moyal }} \\
{\left[A_{\mu}(x), \Omega(x)\right] } & \rightarrow\left\{\mathcal{A}_{\mu}\left(x, z_{1}, z_{2}\right), \Omega\left(x, z_{1}, z_{2}\right)\right\}_{\text {Moyal }}
\end{aligned}
$$

where the brackets are the symmetrised Moyal brackets[17] with respect to the operators $z_{1}$ and $z_{2}$ and the action becomes:

$$
\int \mathrm{d}^{4} x \operatorname{Tr}\left(F_{\mu \nu}(x) F^{\mu v}(x)\right) \rightarrow \int \mathrm{d}^{4} x \mathrm{~d} z_{1} \mathrm{~d} z_{2} \mathcal{F}_{\mu \nu}\left(x, z_{1}, z_{2}\right) * \mathcal{F}^{\mu v}\left(x, z_{1}, z_{2}\right)
$$

with an appropriately defined *-product.

\subsection{A fuzzy torus}

The case of the fuzzy torus is even simpler. For the sphere we had isolated inside $S U(N)$ an $S U(2)$ subgroup and express all the $S U(N)$ generators in terms of the three generators of $S U(2)$. For the torus we isolate a quantum $U(1) \times U(1)$. Let us take first $N$ odd (a similar construction applies to $N$ even) and let $\omega$ be the $N$ th root of unity: $\omega=\mathrm{e}^{4 \pi \mathrm{i} / N}$. We define the two matrices:

$$
g=\left(\begin{array}{ccccc}
1 & 0 & 0 & \ldots & 0 \\
0 & \omega & 0 & \ldots & 0 \\
0 & 0 & \omega^{2} & \ldots & 0 \\
. & . & . & . & 0 \\
. & . & . & . & 0 \\
. & . & . & . & 0 \\
0 & 0 & 0 & \ldots & \omega^{N-1}
\end{array}\right) ; h=\left(\begin{array}{ccccc}
0 & 1 & 0 & \ldots & 0 \\
0 & 0 & 1 & \ldots & 0 \\
. & . & . & . & . \\
. & . & . & . & . \\
. & . & . & . & . \\
0 & 0 & 0 & \ldots & 1 \\
1 & 0 & 0 & \ldots & 0
\end{array}\right)
$$

They satisfy quantum group commutation relations:

$$
g^{N}=h^{N}=1 ; h g=\omega g h
$$

We can use the integer $\bmod N$ powers of these matrices to express the $S U(N)$ generators:

$$
\begin{gathered}
S_{m_{1}, m_{2}}=\omega^{m_{1} m_{2} / 2} g^{m_{1}} h^{m_{2}} \quad ; \quad S_{m_{1}, m_{2}}^{\dagger}=S_{-m_{1},-m_{2}} \\
{\left[S_{\mathbf{m}}, S_{\mathbf{n}}\right]=2 \mathrm{i} \sin \left(\frac{2 \pi}{N} \mathbf{m} \times \mathbf{n}\right) S_{\mathbf{m}+\mathbf{n}}}
\end{gathered}
$$

with $\mathbf{n}=\left(n_{1}, n_{2}\right)$ and $\mathbf{n} \times \mathbf{m}=n_{1} m_{2}-m_{1} n_{2}$. We can show[18] that the algebra (20) is indeed equivalent to that of $S U(N)$ and at the limit $N \rightarrow \infty$ it becomes the algebra of the area preserving diffeomorphisms of a 2-dimensional torus. This connection between $S U(N)$ and [SDiff(T2)] can be made explicit by choosing a pair of variables forming local symplectic coordinates on the torus, for 
example, the angles $z_{1}$ and $z_{2}$ of the two circles, and expanding all functions on the torus on the basis of the eigenfunctions of the laplacian:

$$
h_{n_{1}, n_{2}}=\exp \left(\mathrm{i} n_{1} z_{1}+2 \pi \mathrm{i} n_{2} z_{2}\right) \quad n_{1}, n_{2} \in \mathbb{Z}
$$

Here we are interested in the fuzzy torus, so we endow $z_{1}$ and $z_{2}$ with the commutation relations of the Heisenberg algebra (13). If we define the corresponding group elements $h$ and $g$, by:

$$
h=\mathrm{e}^{\mathrm{i} z_{1}} \quad g=\mathrm{e}^{-2 \mathrm{i} \pi z_{2}}
$$

we can prove again the equivalence:

$$
\left[z_{1}, z_{2}\right]=\frac{2 i}{N} \Leftrightarrow h g=\omega g h
$$

for the set of group elements $h^{n_{1}}$ and $g^{n_{2}}$ with $n_{1}$ and $n_{2}$ integers $\bmod N$. Note that the later imply the algebra of $S U(N)$. The generators of the Heisenberg algebra $z_{i}$ and the group elements $h$ and $g$ are infinite dimensional operators, but we can represent the $S U(N)$ algebra by the finite dimensional ones (17) and (19). They form a discrete subgroup of the Heisenberg group and they have been used to construct quantum mechanics on a discrete phase space[19]. In this case, we can define two new operators $\hat{q}$ and $\hat{p}$, the first being the position operator on the discrete configuration space and the second its finite Fourier transform. They can be represented by $N \times N$ matrices, but, obviously, they do not satisfy anymore the Heisenberg algebra[20].

As we found for the sphere, the Moyal bracket can be defined by symmetrising in $z_{1}$ and $z_{2}$, in which case only odd powers of $1 / N$ appear. The *-product can be written as

$$
f(z) * g(z)=\left.\exp \left(\mathrm{i} \xi \epsilon_{i j} \partial_{z}^{i} \partial_{w}^{j}\right) f(z) g(w)\right|_{w=z}
$$

with $z=\left(z_{1}, z_{2}\right)$ and $\xi=2 / N$. The Yang-Mills action can be written again in the form of equations (14) and (16) and, as before for the sphere, this equivalence is exact at any order in the $1 / N$ expansion.

\section{Non-Commutative Geometry $\Rightarrow$ Gauge Theories}

In the past decades we have learned that all fundamental interactions are described by gauge theories. Gauge transformations are of two sorts:

- Diffeomorphisms in space-time.

- Internal symmetry transformations which apply on some internal space. However, the parameters of these transformations are functions of the space-time point, but not of the point of the internal space. The latter is fixed and does not participate in the dynamics.

Question: Is there a space on which internal symmetry transformations act as diffeomorphisms?

Answer: Yes, but it is a space with non-commutative geometry. A space defined by an algebra of matrix-valued functions

This approach has been developed by Alain Connes and collaborators. Some recent references are listed below[21]. The quantum mechanical non-commutativity involves the introduction of a deformation parameter, to wit $\hbar$, which defines the phase space commutation relations among position and momentum operators for every degree of freedom. The goal here is to extend this notion of non commutativity using mathematical ideas from spectral theory.

The construction involves A fundamental spectral triplet:

Given a spin manifold $M$, the triplet consists of: 
- A Hilbert space $\mathcal{H}$

- An algebra of functions $\mathcal{A}$ which are $C^{\infty}(M)$. $\mathcal{A}$ generalises the notion of space.

- The Dirac operator $\mathcal{D}$, which plays the role of the inverse of distance. (If we ignore gravity, $\mathcal{D}$ can be replaced by the chirality operator)

To this basic triplet we can add decorations, such as chirality, $C P T$, etc.

Although these assumptions sound rather weak, the conditions imposed by spectral theory are quite powerful and restrict the possible choices of $\mathcal{A}$. In particular, the gauge algebras of the Standard Model appear together with General Relativity. Although, to my opinion, the technical part of this construction is not yet fully developed, several physical results may emerge naturally. An incomplete list includes:

- I mentioned already the special role of the Brout-Englert-Higgs scalar as the distance between different branes in the phase with spontaneously broken symmetry.

- This approach may provide the way we are all looking for to unify gauge theories and Gravity.

- It may give some predictions for the Standard Model parameters. An example was a relation between the masses of the vector and the scalar bosons of the electroweak theory. In the traditional framework of renormalisable perturbation theory such a prediction is impossible because there is no zero in the corresponding $\beta$-function[22].

- It may offer a new insight for the mysteries of dark matter and dark energy.

\section{Conclusions and remarks}

The first conclusion is that non-commutative geometry has come to stay! It is part of gauge theories. Whether it will turn out to be convenient for us to use, is still questionable. It will depend on our ability to simplify the mathematics sufficiently, or to master them deeply, in order to get new insights.

Quite independently, let me point out that the spectacular accuracy reached by experiments, as well as theoretical calculations, has made particle physics a precision science. Therefore, "approximate" theories are no more sufficient. A discrepancy by a few percent implies that we do not have the right theory! This strongly restricts the possible ways to go beyond the Standard Model.

On the other hand, the completion of the Standard Model strongly indicates that new and exciting Physics is around the corner. But for the moment, we see no corner! Could non-commutative geometry show the way out of this dilemma?

\section{References}

[1] E.G. Floratos and J. Iliopoulos, Phys. Lett. B201, 237 (1988); E.G. Floratos, J. Iliopoulos and G. Tiktopoulos, Phys. Lett. B217, 285 (1989); E.G. Floratos and J. Iliopoulos, Phys. Lett. B632, 566 (2006)

[2] Letter of Heisenberg to Peierls (1930), Wolfgang Pauli, Scientific Correspondence, Vol. II, p. 15. (Ed. Karl von Meyenn, Springer-Verlag (1985))

[3] Letter of Pauli to Bohr (1947), Wolfgang Pauli, Scientific Correspondence, Vol. II, p. 414. (Ed. Karl von Meyenn, Springer-Verlag (1985))

[4] Letter of Pauli to Oppenheimer (1946), Wolfgang Pauli, Scientific Correspondence, Vol. III, p. 380. (Ed. Karl von Meyenn, Springer-Verlag (1993))

[5] H.S. Snyder, Phys. Rev. 71, 38 (1947). Apparently Snyder had completed this work as early as 1943.

[6] E.D. Courant, M.S. Livingston and H.S. Snyder, Phys. Rev. 88, 1190 (1952)

[7] L.D. Landau, Z. Phys. 64, 629 (1930) 
[8] R. Peierls, Z. Phys. 80, 763 (1933)

[9] For a review, see A. Connes, "Non-Commutative Geometry and Physics", Les Houches summer school, 1992

[10] N. Seiberg and E. Witten, JHEP 09, 032 (1999)

[11] See, for example, A.H. Chamseddine and A. Connes, Fortsch. Phys. 58, 553 (2010); A.H. Chamseddine, A. Connes and V. Mukhanov, JHEP 1412, 098 (2014)

[12] For some reviews see: J. Wess, "Non abelian gauge theories on non-commutative spaces", Proceedings, SUSY02; J. Madore, "An Introduction to non-Commutative Differential Geometry and Physical applications", Cambridge, UK, University Press, 2000; M. R. Douglas and N. A. Nekrasov, Rev. Mod. Phys. 73, 977 (2001); J. Wess, "Gauge theories beyond gauge theories", Fortsch. Phys. 49, 377 (2001); J. Wess, "Gauge theories on non-commutative spaces", Lect. Notes Phys. 616, 320 (2003); R. Jackiw, "Physical instances of noncommuting coordinates", hep-th/0110057; B. Zumino, "Deformation quantisation of nonabelian gauge groups", Proceedings, SUGRA 20; R.J. Szabo, Phys. Rep. 378, 207 (2003)

[13] J. Hoppe, MIT-PhD Thesis, 1982

[14] D. B. Fairlie, P. Fletcher and C. K. Zachos, Phys. Lett. B218, 203 (1989)

[15] B. Konstant, Am. Journ. of Math. 81, 4, 973 (1959)

[16] T. Holstein and H. Primakoff, Phys. Rev. 58, 1098 (1940)

[17] J. Moyal, Proc. Camb. Phil. Soc. 45, 99 (1949)

[18] D. B. Fairlie and C. K. Zachos, Phys. Lett. B224, 101 (1989)

[19] J. Schwinger, "Quantum Mechanics", Springer Verlag, 2003; see also G. G. Athanasiu, E. G. Floratos and S. Nicolis, J. Phys. A Math. Gen. 31, L665 (1998) and references therein.

[20] E. G. Floratos and G. K. Leontaris, Phys.Lett B412, 35 (1997)

[21] Ali H. Chamseddine and Alain Connes: "Why the Standard Model", arXiv:0706.3688 [hep-th]; Ali H. Chamseddine, Alain Connes, and Viatcheslav Mukhanov: "Geometry and the Quantum: Basics", arXiv:1411.0977 [hep-th]; Ali H. Chamseddine, Alain Connes, and Viatcheslav Mukhanov: "Quanta of Geometry: Noncommutative Aspects", arXiv:1409.2471 [hep-th]

[22] J. Iliopoulos: "Can we predict the value of the Higgs mass?", arXiv:0603146 [hep-ph]; S. Heinemeyer, M. Mondragon, N. Tracas and G. Zoupanos: "Reduction of the Parameters in MSSM", arXiv:1712.02729 [hep-ph]; D. Gavriil, G. Manolakos, G. Orfanidis and G. Zoupanos: Fortsch.Phys. 63, 442 (2015) 